\title{
Deconjugated Urinary Metanephrine, Normetanephrine and 3-Methoxytyramine in Laboratory Diagnosis of Pheochromocytoma and Paraganglioma
}

\author{
R. BÍLEK ${ }^{1}$, T. ZELINKA ${ }^{2}$, P. VLČEK ${ }^{3}$, J. DUŠKOVÁ ${ }^{4}$, D. MICHALSKÝ ${ }^{5}$, K. NOVÁK $^{6}$, \\ J. BEŠŤÁK ${ }^{1}$, J. WIDIMSKÝ Jr. ${ }^{2}$
}

${ }^{1}$ Institute of Endocrinology, Prague, Czech Republic, ${ }^{2}$ Center for Hypertension, Third Medical Department - Department of Endocrinology and Metabolism, First Faculty of Medicine, Charles University and General University Hospital, Prague, Czech Republic, ${ }^{3}$ Department of Nuclear Medicine and Endocrinology, Faculty Hospital Motol and Second Faculty of Medicine, Charles University, Prague, Czech Republic, ${ }^{4}$ Czech Republic Institute of Pathology, First Faculty of Medicine, Charles University in Prague and General University Hospital, Prague, Czech Republic, ${ }^{5}$ First Department of Surgery - Department of Abdominal, Thoracic Surgery and Traumatology, First Faculty of Medicine, Charles University and General University Hospital, Prague, Czech Republic, ${ }^{6}$ Department of Urology, First Faculty of Medicine, Charles University and General University Hospital, Prague, Czech Republic

Received June 17, 2015

Accepted July 2, 2015

\section{Summary}

This work discusses the clinical performance of deconjugated metanephrine (MN), normetanephrine (NMN) and 3-methoxytyramine (3MT) determined in the basal first morning urine using a chromatographic method with electrochemical detection for the clinical diagnosis of pheochromocytoma (PHEO) and paraganglioma (PGL). Urine samples were collected from 44 patients (36 with PHEO, 8 with PGL) aged 54+/-17 (20-78) years (22 females, 22 males). A sampling of biological materials was performed preoperatively and about one week, six months and one year after adrenal gland surgery. The control group consisted of $34 \mathrm{PHEO} / \mathrm{PGL}$ patients more than 4 months after adrenal gland surgery. All subjects in the control group were without a diagnosis of PHEO or PGL. Clinical sensitivity was $55 \%$ for MN, $64 \%$ for NMN, $80 \%$ for combination of both MN and NMN, and only $23 \%$ for 3TM. Clinical specificity calculated from the control group was $93 \%$ for MN, $95 \%$ for NMN, $95 \%$ for the combination MN and NMN, and $97 \%$ for 3TM. Cut-off values for deconjugated metanephrines in the basal urine were $310(\mathrm{MN})$, 690 (NMN) and $250 \mu \mathrm{g} / \mathrm{l}$ (3MT). Chromatographic determination of deconjugated urinary metanephrines, which is simple without the necessity of special laboratory material, can serve for the screening of PHEO or PGL patients. Urine NMN and 3MT exerts an association to malignity, and all markers are associated with tumor mass. However, the principal laboratory diagnosis of PHEO or PGL must be based on plasma-free metanephrines and plasma chromogranin A with better performance in the laboratory diagnosis of PHEO or PGL.

\section{Key words \\ Urine - Deconjugated metanephrine - Deconjugated normetanephrine - Deconjugated 3-methoxytyramine • Chromatography - Electrochemical detection • Pheochromocytoma • Paraganglioma}

\section{Corresponding author}

R. Bilek, Institute of Endocrinology, Narodni 8, 11694 Prague 1, Czech Republic. Fax: +420 224905325. E-mail: rbilek@endo.cz

\section{Introduction}

Pheochromocytoma (PHEO) or paraganglioma (PGL) are rare tumors associated with neuroendocrine chromaffin cells usually found in the adrenal medulla and other ganglia of the nervous system (Tischler 2006). PHEO is a tumor arising from adrenomedullary 
chromaffin cells that commonly produces catecholamines and it is associated with adrenal localization in $80-85 \%$ of patients. PGL is a neuroendocrine tumor derived from extra-adrenal chromaffin cells of the sympathetic paravertebral ganglia (thorax, abdomen, pelvis) producing catecholamines, or parasympathetic ganglia located in the neck and at the base of the skull, which do not produce catecholamines (Chen et al. 2010, Lenders et al. 2014). Extra-adrenal occurrence of sympathetic and parasympathetic PGL is observed in 10-20\% patients (Chen et al. 2010, van Berkel et al. 2014). In this study we have used PPGL as a general term for both PHEO and PGL. Approximately $10 \%$ of the PHEOs and $20 \%$ of the PGLs are malignant with poor survival (Andersen et al. 2011). Up to $30 \%$ of PPGL patients have recognizable germline mutations including multiple endocrine neoplasia type 2A (MEN2A) or 2B (MEN2B), von Recklinghausen neurofibromatosis type 1 (NF1), von Hippel-Lindau syndrome (VHL) and succinate dehydrogenase complex - subunits A, B, C, D (SDHx) syndrome (Mazzaglia 2012). Since 1990, 14 different PPGL susceptibility genes have been reported (Lenders et al. 2014). Mainly mutations in the tumour suppressor gene von Hippel-Lindau (VHL) and the mitochondrial succinate dehydrogenase enzyme complex subunit B $(S D H B)$ are associated with malignancy (Andersen et al. 2011).

The genetic diversity commonly associated with PPGLs is based on the noradrenergic phenotype of VHL and SDHx or on the adrenergic phenotype characteristic for MEN2A, MEN2B and for NF1 (van Berkel et al. 2014). In noradrenergic phenotype the biosynthesis of the enzyme phenylethanolamine-N-methyltransferase converting norepinephrine to epinephrine is downregulated at both the mRNA and protein level in sympathetic or parasympathetic paragangliomas (Gouzmann et al. 2015). Increased production of norepinephrine results in an elevated concentration of normetanephrine in circulation after the conversion of norepinephrine to normetanephrine by the enzyme catechol O-methyltransferase. The epinephrine and metanephrine are usually within the normal reference range in noradrenergic PGLs. In the adrenergic phenotype, the phenylethanolamine-N-methyltransferase presented in the adrenal gland converts norepinephrine to epinephrine, which is further converted by the catechol O-methyltransferase to metanephrine. The adrenergic phenotype is typical with an elevated concentration of epinephrine and secondary metanephrine with varying amounts of norepinephrine and normetanephrine in the circulation (van Berkel et al. 2014). A lack of the catechol O-methyltransferase in sympathetic nerves, the major site of initial norepinephrine metabolism, means that the O-methylated metabolites are relatively specific markers of chromaffin tumors. These metabolites are produced continuously within tumors by a process that is independent of exocytotic catecholamine release, which for some tumors occurs at low rates or is episodic in nature (Lenders et al. 2014). In addition, $70 \%$ of patients with SDHB and SDHD mutations increase 3-methoxytyramine (dopamine) production (Eisenhofer et al. 2011) due to the absence of tyrosine hydroxylase, the enzyme that catalyses the initial and rate-limiting step in catecholamine biosynthesis (van Berkel et al. 2014). Dopaminergic phenotype can be a good indicator of metastatic disease (Eisenhofer et al. 2012, Fliedner et al. 2010). Sympathetic PPGL usually produce significant amounts of catecholamines, whereas parasympathetic PGL can be endocrinologically inactive (Lenders et al. 2005). It was shown (Eisenhofer et al. 2011) that patients with NF1 and MEN2 can be discriminated from those with VHL, SDHB and SDHD mutations in $100 \%$ cases by the combined analysis of MN, NMN and 3MT. Within this subgroup of VHL, SDHB or SDHD patients, measurements of plasma $3 \mathrm{MT}$ discriminated patients with SDHB and SDHD mutations from those with VHL mutations in $78 \%$ of cases. The histological evaluation of primary PPGL tumors is based on PASS scoring scale. A value less than 4 out of 20 points supports the benign character of the tumor (Thomson 2002).

High-performance liquid chromatography (HPLC) continues to be the dominant analytical method for biogenic amine quantitation. The most widely used method for the determination of catecholamines and metanephrines that have been extracted from urine and separated by HPLC is electrochemical detection (ECD). However, the use of more sensitive liquid chromatography with tandem mass spectrometry (LC-MS/MS) is becoming more popular (Whiting and Doogue 2009). Simultaneous measurements of urinary free O-methylated metabolites of catecholamines by the liquid chromatographic tandem-mass spectrometric method provide potential advantages over urinary deconjugated metanephrines for the diagnosis of PPGLs with analytical sensitivity $1.2 \mathrm{nmol} / 1$ (Peitsch et al. 2013). The Endocrine Society (Lenders et al. 2014) recommends that the initial screening for PPGLs include 
measurements of plasma-free metanephrines or urine fractionated metanephrines using liquid chromatography with mass spectrometric or electrochemical detection methods. LC-MS/MS offers numerous advantages over other analytical methods and is the method of choice when measurements include methoxytyramine (Eisenhofer and Peitzsch 2014). LC-MS/MS limit of quantification under $75 \mathrm{pmol} / \mathrm{l}$ was achieved for $\mathrm{MN}$, NMN and 3-methoxytyramine using only $150 \mu \mathrm{l}$ of plasma samples (Adaway et al. 2015).

Prior to chromatography, a pre-analytical clean-up step is required to extract and sometimes to concentrate the metanephrines from urine. Metanephrines are metabolised by sulfatation and excreted in the urine predominantly as sulphated metabolites (Eisenhofer et al. 2004). Urinary metanephrines are usually measured after deconjugation, where the results reflect the total concentration of metanephrines in urine. An acid hydrolysis of urinary metanephrines, where the biogenic amines are protonated as cations, and can be isolated using solid-phase extraction (SPE) based on both cation-exchange and anion-exchange chromatography to separate metanephrines from catecholamines and other interfering compounds, is nearly always included to release the conjugated forms, which are the major molecular species present (Whiting and Doogue 2009). New column technology has been developed with smaller particle sizes and with increased resolving power based on reverse-phase chromatography with modifications to allow retention and separation of polar biogenic amines without using ion-pairing reagents.

The plasma test offers advantages over the urine test, although it is rarely implemented correctly, rendering the urine test preferable for mainstream use (Eisenhofer and Peitzsch 2014). Urinary collection provides an integral measurement over $24 \mathrm{~h}$, but is potentially incomplete and is inconvenient for patients (van Berkel et al. 2014). Several pre-analytical factors, such as exercise, posture, food with substantial quantities of biogenic amines, stress, hypoglycemia and medications may affect test results (van Berkel et al. 2014).

In this study we presented our experiences with high performance liquid chromatography with electrochemical detection (HPLC-ECD) for determination of urine deconjugated $\mathrm{MN}, \mathrm{NMN}$ and 3MT in groups of patients who suffered of PPGLs.

\section{Materials and Methods}

\section{The group of patients}

Included in the study were the group of PPGL patients and the control group of the same patients more than 4 months after adrenalectomy without diagnosis of PPGL in this time. Patients with pheochromocytoma or paraganglioma consisted of 44 patients aged $54+/-17$ (20-78) years, 22 females aged 60+/-16 (28-78) years, 22 males aged 49+/-17 (20-76) years. Pheochromocytoma occurred in 36 patients, paraganglioma in 8 patients. Adrenergic phenotype of disease was observed in 23 patients (1 MEN2A+MTC, 2 NF1, 20 PHEO), noradrenergic phenotype was found in 21 patients (11 PHEO, 7 PGL, 1 SDHD, 2 VHL).

A sampling of biological materials (first morning urine) was performed preoperatively and about one week, six months and one year after surgery. All four collections were conducted in 24 patients (17 PHEO, 2 PGL, 2 NF1, 1 VHL, 1 SDHD, 1 MEN2a+MTC), 3 collections in 8 patients (5 PHEO, 2 PGL, 1 VHL), 2 collections in 8 patients (5 PHEO, 2 PGL, 1 VHL), and 1 collection taking before surgery was conducted in 4 patients (1 PHEO, 3 PGL).

The control group consisted of 34 PHEO or PGL patients more than 4 months after adrenalectomy (24 PHEO, 3 PGL, 2 NF1, 3 VHL, 1 SDHD, 1 MEN2a+MTC).

\section{Laboratory examination}

$\mathrm{MN}, \mathrm{NMN}$ and $3 \mathrm{MT}$ were determined in the basal first morning urine using a solid-phase extraction (SPE) after deconjugation of metanephrines by acid hydrolysis of metanephrine-sulphates. The deconjugated MN, NMN and 3MT were determined by HPLC-ECD.

Acid hydrolysis: $100 \mu \mathrm{l}$ of urine (or calibrators) together with $100 \mu \mathrm{l}$ of internal standard consisting of $2 \mu \mathrm{mol} / \mathrm{l}$ 4-hydroxy-3-methoxybenzylamine hydrochloride (HMBA) in $100 \mu \mathrm{l}$ of $95 \mu \mathrm{mol} / 1 \mathrm{CH}_{3} \mathrm{COOH}$ : $\mathrm{CH}_{3} \mathrm{CN}=200: 0.01$ and $50 \mu \mathrm{l} 1 \mathrm{~mol} / 1 \mathrm{HCl}$ were heated for $20 \mathrm{~min}$ at $90{ }^{\circ} \mathrm{C}$.

SPE with Separon HEMA 1000 CM (Tessek, Prague, Czech Republic, $d_{\mathrm{p}}=60 \mu \mathrm{m}$, weakly acidic cation exchanger): $50 \mu \mathrm{l}(=20 \mu \mathrm{l}$ of urine with 40 pmol of HMBA) of hydrolyzate and $200 \mu \mathrm{l}$ of borate buffer (pH 6.5; $3 \mathrm{~g}$ of $\left(\mathrm{NH}_{4}\right) \mathrm{B}_{5} \mathrm{O}_{8} .8 \mathrm{H}_{2} \mathrm{O}$ and $2 \mathrm{~g}$ of boric acid in $100 \mathrm{ml}$ of water) were applied to filter tip with sorbent, washed with $500 \mu \mathrm{l}$ of water and eluted with $500 \mu \mathrm{l}$ of $3 \mathrm{~mol} / 1 \mathrm{NH}_{4} \mathrm{OH}$. 
SPE with Dowex 1x2 chloride form (SigmaAldrich s.r.o., Prague, Czech Republic, 200-400 mesh, strong anion exchanger): $500 \mu$ of eluate from Separon HEMA $1000 \mathrm{CM}(=20 \mu \mathrm{l}$ of urine and $40 \mathrm{pmol}$ of HMBA) were applied to filter tip with sorbent, washed with $500 \mu \mathrm{l}$ of water and eluted with $250 \mu \mathrm{l}$ of $50 \mathrm{mmol} / \mathrm{l}$ citrate buffer ( $\mathrm{pH} 2.95)$.

HPLC (Double piston pump Knauer 1050, Wissenschaftliche Gerätebau Dr. Ing. Herbert Knauer $\mathrm{GmbH}$, Berlin, Germany): $10 \mu \mathrm{l}$ of eluate $(=0.4 \mu \mathrm{l}$ of urine and 0.8 pmol of HMBA) was injected to the stationary phase Synergi $4 \mu \mathrm{m}$ Polar-RP $80 \mathrm{~A}$ (Phenomenex, Torrance, CA, USA) in 150 x $3 \mathrm{~mm}$ stainless steel column. Mobile phase consisted of acetonitrile: water containing $0.17 \mathrm{mmol} / \mathrm{l}$ EDTA, $2 \mathrm{mmol} / \mathrm{l} \mathrm{KCl}, 40 \mathrm{mmol} / 1 \mathrm{NaH}_{2} \mathrm{PO}_{4} .2 \mathrm{H}_{2} \mathrm{O}, 10 \mathrm{mmol} / \mathrm{l}$ $\mathrm{H}_{3} \mathrm{PO}_{4}, 1 \mathrm{mmol} / 1$ 1-octanesulfonic acid sodium salt and $0.06 \mathrm{mmol} / 1 \quad 1,4,7,10,13,16$-hexaoxacyclooctadecane $=80: 920$. The isocratic elution was used with the flow rate $0.6 \mathrm{ml} / \mathrm{min}$. Separated metanephrines were detected using amperometric detector Decade II (Antec, Leyden, The Netherlands) with working voltage $+0.65 \mathrm{~V}$, current range $2 \mathrm{nA}$.

\section{Statistics and calculations}

All statistical calculations were made using the computer program NCSS 2004 (Number Cruncher Statistical Systems, Kayville, Utah, USA). The individual points in the time series of the CGA, MN, and NMN examination were interpolated after an inverse or logarithmic transformation by the Newton polynomial programmed up to the 12 th degree using our own program working in Lotus 1-2-3 of the IBM Corporation, USA. Individual curves were counted for all patients with 3 and 4 determinations of CGA, MN, and NMN during more than the annual monitoring of their condition.

\section{Results}

The curves modeling the course of changes in $\mathrm{MN}, \mathrm{NMN}$ and $3 \mathrm{MT}$ concentrations during the 75 weeks of monitoring PPGL patients following adrenal gland operations are based on 3 to 4 determinations of MN, NMN and 3MT. These curves along with specific values of MN, NMN and 3MT are shown in Figures 1, 2 and 3. For all patients diagnosed with PPGL, the nature of the illness was differentiated in the figures by solid symbols given in the legend of the Figures. These were patients suffering from PHEO, PGL, SDHD, NF1, VHL and MEN2A with MTC syndromes. The curves were modelled so that the concentrations of monitored analytes before the operation were transferred to the $\mathrm{Y}$ axis to the 0 time corresponding to the operation on the adrenal gland. The reference range is defined by a transparent rectangle on the curves. The course of the curves in a given reference range was recorded in the part of the Figures labelled with the letter B. The clinical sensitivity of the individual tests for determining MN, NMN and 3MT is well evident in this part. The mean curve of the individual analytes consists of patient values for whom concentrations at time 0 were greater than the cut-off value for $\mathrm{MN}$, NMN or 3MT is depicted on the Figures in part C. A dashed curve shown here consists of the mean curve to which twice the value of the standard deviation was added. The maximum of this dashed curve in time more than 10 weeks after surgery of adrenal gland corresponds with cut-off value which is upper part of reference range. This curve tells us with $95 \%$ probability when the concentration of the individual analytes will return to the normal reference range following the operation.

Figure 1 describes the changes in $\mathrm{MN}$ concentration in a given time period and it is evident (Fig. 1A, 1B) that the clinical sensitivity is relatively low for patients with PPGL (55\%), the clinical specificity is $93 \%$ and Figure $1 \mathrm{C}$ shows the reference range with the cut-off value $310 \mu \mathrm{g} / \mathrm{l}$. MN returns to a normal reference range in an average of 2.5 weeks (Fig. 1C).

Figure 2 describes changes in NMN concentration in a given time period and it is evident (Fig. 2A, 2B) that clinical sensitivity reaches only $64 \%$ in PPGL patients. The clinical sensitivity is higher than the clinical sensitivity for $\mathrm{MN}$, but it is insufficient for laboratory diagnostics of PPGL patients. The combined sensitivity of $\mathrm{MN}$ and NMN corresponds to a value of $80 \%$. As with $\mathrm{MN}$, clinical specificity reached $95 \%$ (Fig. 2A) and NMN returned to a reference interval with a cut-off value of $690 \mu \mathrm{g} / \mathrm{l}$ in 2.4 weeks on average.

Figure 3 describes changes in $3 \mathrm{MT}$ concentration in a given time period and it is evident (Fig. 3A, 3B) that clinical sensitivity reaches only $23 \%$ and $3 \mathrm{MT}$ is completely inappropriate for general laboratory diagnostics of PPGL patients. Clinical specificity was in the case of $3 \mathrm{MT}$ equal to $97 \%$. $3 \mathrm{MT}$ returned to the normal reference range with a cut-off value of $250 \mu \mathrm{g} / \mathrm{l}$ in 15 weeks on average. 

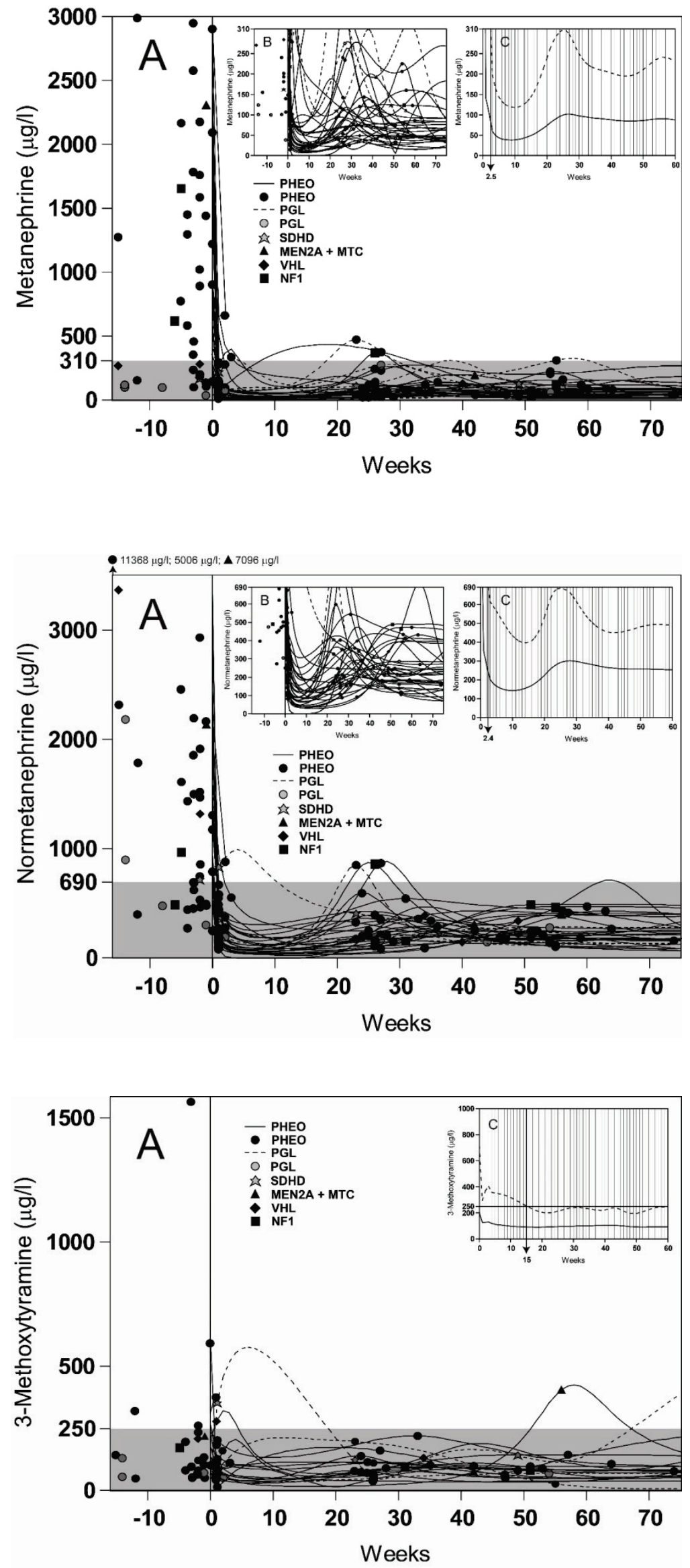

Fig. 1. Shown in part $\mathbf{A}$ are the results of determining metanephrine (MN) with specific values determined before the operation and after the operation using symbols describing the type of syndrome of pheochromocytoma (PHEO) or paraganglioma (PGL) patients. The values are linked by the curves calculated using a Newton polynomial of the $12^{\text {th }}$ degree and a transparent rectangle indicates the reference range. The values and curves in part B correspond to the given reference range. The mean $\mathrm{MN}$ curve taken from patient values for whom concentrations at time 0 were greater than the cut-off value for $M N$ is depicted on the figures in part $\mathbf{C}$. A dashed curve shown here consists of the mean curve to which twice the value of the standard deviation (SD) was added. PHEO, pheochromocytoma; PGL, paraganglioma; MEN, multiple endocrine neoplasia; MTC, medullary thyroid cancer; NF1, von Recklinghausen neurofibromatosis type 1 ; VHL, von Hippel-Lindau syndrome; SDHD, succinate dehydrogenase complex - subunit D syndrome.

Fig. 2. Shown in part $\mathbf{A}$ are the results of determining normetanephrine (NMN) with specific values determined before the operation and after the operation using symbols describing the type of syndrome of pheochromocytoma (PHEO) or paraganglioma (PGL) patients. The values are linked by the curves calculated using a Newton polynomial of the $12^{\text {th }}$ degree and a transparent rectangle indicates the reference range. The values and curves in part B correspond to the given reference range. The mean NMN curve taken from patient values for whom concentrations at time 0 were greater than the cut-off value for NMN is depicted on the figures in part C. A dashed curve shown here consists of the mean curve to which twice the value of the standard deviation (SD) was added. PHEO, pheochromocytoma; PGL, paraganglioma; MEN, multiple endocrine neoplasia; MTC, medullary thyroid cancer; NF1, von Recklinghausen neurofibromatosis type 1 ; $\mathrm{VHL}$, von Hippel-Lindau syndrome; SDHD, succinate dehydrogenase complex - subunit $D$ syndrome.

Fig. 3. Shown in part $\mathbf{A}$ are the results of determining 3-methoxytyramine (3MT) with specific values determined before the operation and after the operation using symbols describing the type of syndrome of PPGL patients. The values are linked by the curves calculated using a Newton polynomial of the $12^{\text {th }}$ degree and a transparent rectangle indicates the reference range. The mean 3MT curve taken from patient values for whom concentrations at time 0 were greater than the cut-off value for 3MT is depicted on the figures in part C. A dashed curve shown here consists of the mean curve to which twice the value of the standard deviation (SD) was added. PHEO, pheochromocytoma; PGL, paraganglioma; MEN, multiple endocrine neoplasia; MTC, medullary thyroid cancer; NF1, von Recklinghausen neurofibromatosis type 1 ; $\mathrm{VHL}$, von Hippel-Lindau syndrome; SDHD, succinate dehydrogenase complex - subunit $D$ syndrome. 
All results concerning clinical sensitivity in patients with a PPGL diagnosis, measured before the adrenal gland operation, are shown in Table 1. Patients are divided into adrenergic and noradrenergic phenotypes and both cut-off values for $\mathrm{MN}, \mathrm{NMN}, 3 \mathrm{MT}$ and their mean values, standard deviations, ranges of measured concentration in first morning urine and the number of PPGL patients diagnosed with a specific disease syndrome are given here. In summary, it can be stated that clinical sensitivity had a value of $55 \%$ for $\mathrm{MN}, 64 \%$ for NMN, $80 \%$ for a combination of $\mathrm{MN}+\mathrm{NMN}$ and $23 \%$ for $3 \mathrm{MT}$.

Table 2 gives all results concerning clinical specificity for both PPGL patients with MN, NMN and $3 \mathrm{MT}$ values that were included in calculations only in the case that they were determined at least 4 months following the adrenal gland operation. This concerned a total of 34 patients. The Table 2 gives both cut-off values for $\mathrm{MN}$, NMN, 3MT and their mean values, standard deviations, ranges of measured concentration in first morning urine and the number of PPGL patients diagnose. Clinical specificity has a value of $93 \%$ for $\mathrm{MN}, 95 \%$ for $\mathrm{NMN}$, $95 \%$ for a combination of MN/NMN and $97 \%$ for $3 \mathrm{MT}$. The mass of the operated tumor correlated to the $\mathrm{MN}$, NMN and 3MT concentration according to the equations: $\log (\operatorname{mass}(\mathrm{g}))=0.1406+0.5845 * \log (\mathrm{MN}(\mu \mathrm{g} / \mathrm{l}))$

$$
\mathrm{p}<0.0001, \mathrm{r}=0.6870
$$

$\log (\operatorname{mass}(\mathrm{g}))=-0.4888+0.7498 * \log (\mathrm{NMN}(\mu \mathrm{g} / \mathrm{l}))$ $\mathrm{p}=0.0022, \mathrm{r}=0.5079$

$\log (\operatorname{mass}(\mathrm{g}))=0.0012+0.7923 * \log (3 \mathrm{MT}(\mu \mathrm{g} / \mathrm{l}))$

$$
\mathrm{p}=0.0002, \mathrm{r}=0.6023
$$

PASS scores correlated only with concentrations NMN and $3 \mathrm{MT}$ according to equations:

$$
\begin{gathered}
\log (\text { PASS })=-0.2393+0.3032 * \log (\mathrm{NMN}(\mu \mathrm{g} / \mathrm{l})) \\
\mathrm{p}=0.0333, \mathrm{r}=0.3417 \\
\log (\text { PASS })=-0.0512+0.3264 * \log (3 \mathrm{MT}(\mu \mathrm{g} / \mathrm{l})) \\
\mathrm{p}=0.0096, \mathrm{r}=0.4097
\end{gathered}
$$

A statistically important correlations between the ascertained AUC (area under curve) logarithmic values of $\mathrm{MN}, \mathrm{NMN}, 3 \mathrm{MT}$ and logarithmic values of the tumor mass or PASS score were not found.

\section{Discussion}

Chromatographic determination of $\mathrm{MN}, \mathrm{NMN}$, 3MT employs a simple and feasible technology for detection of PPGL diseases. The problem is that during initial diagnostic workup in patients with suspected PPGL tumors a single test with very high negative predictive value is preferred. The increase of clinical sensitivity can lower the clinical specificity (van Berkel et al. 2014). In the case of $\mathrm{MN}$, the predictive value of the test failed from a clinical sensitivity standpoint for patients with noradrenergic phenotype. By contrast, there can be a problem with NMN for patients with adrenergic phenotype. In the case of PPGL patients, it is therefore very important to conduct a parallel determination of both MN and NMN. In our group of patients, determination of clinical sensitivity via $3 \mathrm{MT}$ was the least successful. Increased concentrations of 3MT can be helpful in the laboratory diagnosis of patients with an endocrinologically inactive PGL nature or in those cases in which dedifferentiation of chromaffin cells no longer allows for the biosynthesis of catecholamines. This type of patient did not occur in the group we observed.

The modeling of the individual curves from 3 to 4 of the values determined over the course of over a year monitoring PPGL patients was complicated. The values had to be inversely or logarithmically transformed so that a Newton polynomial of the $12^{\text {th }}$ degree could be applied to modeling the course via a program developed in our laboratory. The result of the modeling enabled the course of change to be predicted, provided information on the used reference ranges and enabled a determination of the mean period of the return of $\mathrm{MN}, \mathrm{NMN}$ and $3 \mathrm{MT}$ to the normal reference range following the adrenal gland operation. This period was less than 3 weeks for MN or NMN, but nearly 15 weeks for 3MT. The AUC (area under) given in Table 2 provides information on the overall biosynthesis of $\mathrm{MN}$, NMN and 3MT during the monitored period. We did not found the statistically significant relationship between mass of removed tumor or PASS score and values of MN, NMN or 3MT. The AUC values should be further examined from a clinical standpoint. Statistically important associations were found for the logarithmic transformation between the mass of the operated tumor and $\mathrm{MN} \quad(\mathrm{p}<0.0001), \mathrm{NMN} \quad(\mathrm{p}=0.0022)$ or $3 \mathrm{MT}$ $(p=0.0002)$. The PASS scores only correlated with NMN $(p=0.0333)$ and $3 \mathrm{MT} \quad(p=0.0096)$ after the logarithmic transformation of values.

Our cut-off values for urinary $\mathrm{MN}, \mathrm{NMN}$ and 3MT were based on basal values measured in the first morning urine. This is a more practical method than measuring the values related to a 24-h urine sample, which for many cases, especially those patients immediately following surgery, is feasible only with difficulties. 


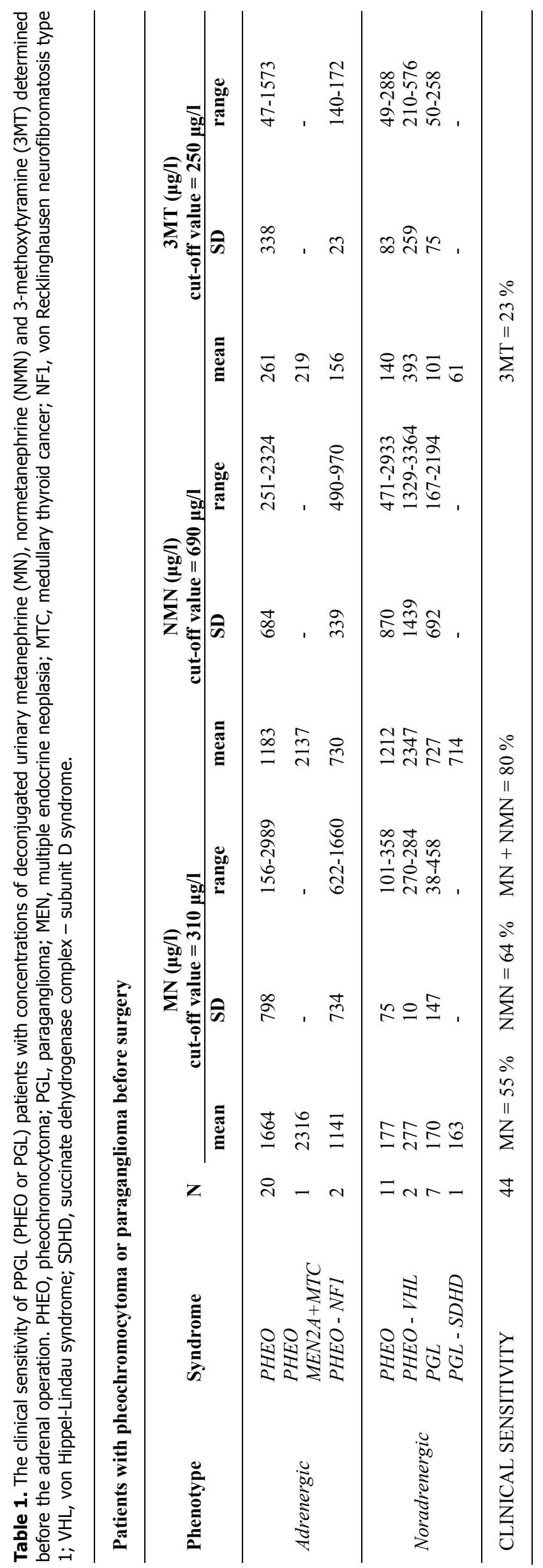




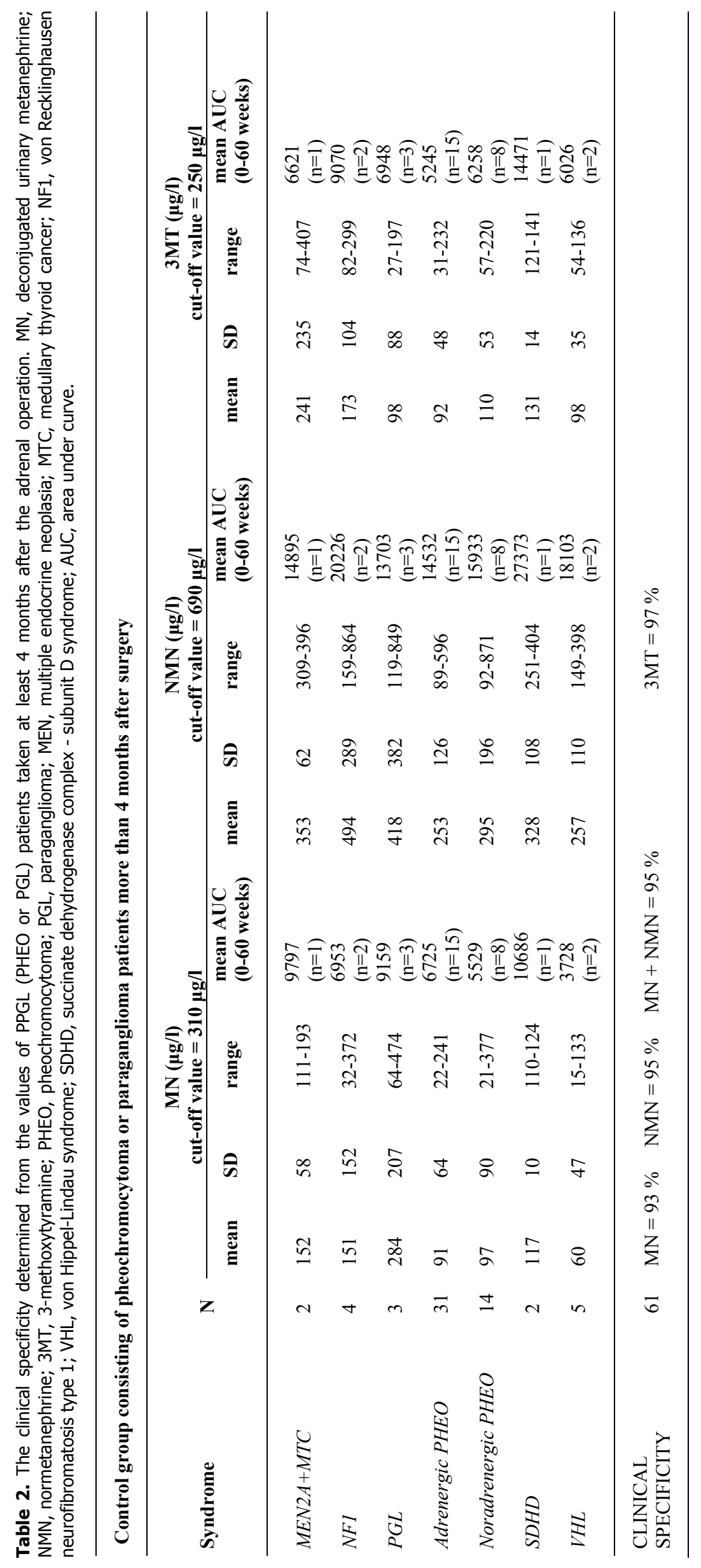


Assuming normal fluid intake, this method does not have to overly increase the uncertainty of the measurement with regard to the clinical interpretation of the results. The cut-off values were set so that maximum specificity is achieved, which, on the other hand, reduces the predictive value for clinical sensitivity. This is another reason why the laboratory diagnosis of PPGL patients must be augmented by measuring free metanephrines and chromogranin A in plasma.

\section{Conclusion}

We can conclude that the determination of deconjugated urine MN, NMN and 3MT using HPLCECD, which is relatively simple without the necessity of special laboratory material, can be an effective markers for screening of PPGLs. Urinary metanephrines exerts association to malignity and tumor mass. One limitation is that their clinical sensitivity and clinical specificity were worse than those parameters determined using radioimmunoassay or chromatographic processes in the plasma. Urinary metaphrines are appropriate after screening PPGL patients following adrenal gland surgery, but their predictive values is not sufficient with enough certainty to draw definitive conclusions regarding clinical sensitivity and specificity. The laboratory diagnosis of PPGL patients must be augmented by measuring plasmafree $\mathrm{MN}$ and NMN. It is also certainly useful in this respect to augment and confirm the laboratory diagnosis via the values of chromogranin A determined by radioimmunoassay in plasma or serum. The advantage of the immunoassay together with HPLC-ECD approach is that these methods are common in a clinical-biochemical laboratory and do not require additional expensive instrumentation.

\section{Conflict of Interest}

There is no conflict of interest.

\section{Acknowledgements}

This work was supported by grant IGA MZ CR NT/12336-4.

\section{References}

ADAWAY JE, PEITZSCH M, KEEVIL BG: A novel method for the measurement of plasma metanephrines using online solid phase extraction-liquid chromatography tandem mass spectrometry. Ann Clin Biochem 52: 361-369, 2015.

ANDERSEN KF, ALTAF R, KRARUP-HANSEN A, KROMANN-ANDERSEN B, HORN T, CHRISTENSEN NJ, HENDEL HW: Malignant pheochromocytomas and paragangliomas - the importance of a multidisciplinary approach. Cancer Treat Rev 37: 111-119, 2011.

CHEN H, SIPPEL RS, O'DORISIO MS, VINIK AI, LLOYD RV, PACAK K: The North American Neuroendocrine Tumor Society consensus guideline for the diagnosis and management of neuroendocrine tumors: pheochromocytoma, paraganglioma, and medullary thyroid cancer. Pancreas 39: 775-783, 2010.

EISENHOFER G, PEITZSCH M: Laboratory evaluation of pheochromocytoma and paraganglioma. Clin Chem 60: 1486-1499, 2014.

EISENHOFER G, KOPIN IJ, GOLDSTEIN DS: Catecholamine metabolism: a contemporary view with implications for physiology and medicine. Pharmacol Rev 56: 331-349, 2004.

EISENHOFER G, LENDERS JW, TIMMERS H, MANNELLI M, GREBE SK, HOFBAUER LC, BORNSTEIN SR, TIEBEL O, ADAMS K, BRATSLAVSKY G, LINEHAN WM, PACAK K: Measurements of plasma methoxytyramine, normetanephrine, and metanephrine as discriminators of different hereditary forms of pheochromocytoma. Clin Chem 57: 411-420, 2011.

EISENHOFER G, LENDERS JW, SIEGERT G, BORNSTEIN SR, FRIBERG P, MILOSEVIC D, MANNELLI M, LINEHAN WM, ADAMS K, TIMMERS HJ, PACAK K: Plasma methoxytyramine: a novel biomarker of metastatic pheochromocytoma and paraganglioma in relation to established risk factors of tumour size, location and SDHB mutation status. Eur J Cancer 48: 1739-1749, 2012.

FLIEDNER SM, LEHNERT H, PACAK K: Metastatic paraganglioma. Semin Oncol 37: 627-637, 2010.

GROUZMANN E, TSCHOPP O, TRIPONEZ F, MATTER M, BILZ S, BRÄNDLE M, DRECHSER T, SIGRIST S, ZULEWSKI H, HENZEN C, FISCHLI S, ABID K: Catecholamine metabolism in paraganglioma and pheochromocytoma: similar tumors in different sites? PLoS One 10: e0125426, 2015.

LENDERS JW, EISENHOFER G, MANNELLI M, PACAK K: Phaeochromocytoma. Lancet 366: 665-675, 2005. 
LENDERS JW, DUH QY, EISENHOFER G, GIMENEZ-ROQUEPLO AP, GREBE SK, MURAD MH, NARUSE M, PACAK K, YOUNG WF JR; ENDOCRINE SOCIETY: Pheochromocytoma and paraganglioma: an endocrine society clinical practice guideline. J Clin Endocrinol Metab 99: 1915-1942, 2014.

MAZZAGLIA PJ: Hereditary pheochromocytoma and paraganglioma. J Surg Oncol 106: 580-585, 2012.

PEITZSCH M, PELZEL D, GLÖCKNER S, PREJBISZ A, FASSNACHT M, BEUSCHLEIN F, JANUSZEWICZ A, SIEGERT G, EISENHOFER G: Simultaneous liquid chromatography tandem mass spectrometric determination of urinary free metanephrines and catecholamines, with comparisons of free and deconjugated metabolites. Clin Chim Acta 418: 50-58, 2013.

THOMSON LDR: Phaeochromocytoma of the adrenal gland scoring scale (PASS) to separate benign from malignant neoplasms. A clinicopathologic and immunophenotypic study of 100 cases. Am J Surg Pathol 26: 551-566, 2002.

TISCHLER AS: Molecular and cellular biology of pheochromocytomas and extra-adrenal paragangliomas. Endocr Pathol 17: 321-328, 2006.

VAN BERKEL A, LENDERS JW, TIMMERS HJ: Diagnosis of endocrine disease: Biochemical diagnosis of phaeochromocytoma and paraganglioma. Eur J Endocrinol 170: R109-R119, 2014.

WHITING MJ, DOOGUE MP: Advances in biochemical screening for phaeochromocytoma using biogenic amines. Clin Biochem Rev 30: 3-17, 2009. 